\title{
Adherence Kinetics of Haemophilus influenzae Type b to Eucaryotic Cells ${ }^{1}$
}

\author{
CHRISTIAN C. PATRICK, ${ }^{2}$ GAIL S. PATRICK, SHELDON L. KAPLAN, JAMES BARRISH, AND \\ EDWARD O. MASON, JR. \\ The Myers Black Infectious Diseases Section, Departments of Pediatrics [C.C.P., G.S.P., S.L.K., E.O.M.], \\ Microbiology and Immunology [E.O.M.], and Pathology [J.B.], Baylor College of Medicine, \\ and Texas Children's Hospital, Houston, Texas 77030
}

\begin{abstract}
Haemophilus influenzae type b (Hib) adhere in vitro to buccal epithelial cells (BEC) via pili (fimbriae). In vivo studies have conflicting lines of evidence concerning the role of pili in adherence. This study characterizes the kinetics of Hib binding to BEC and compares pilus versus nonpilus adherence. Adherence was assessed using radioactive, immunofluorescence, and electron microscopic techniques. Paired Hib strains were obtained from three children with Hib meningitis; piliated Hib were isolated from the nasopharynx and nonpiliated isolates from the cerebrospinal fluid. Piliated strains adhered avidly to BEC after a 1-h incubation, whereas nonpiliated strains adhered poorly $(p=0.002)$. Kinetic analysis revealed increased adherence to BEC by piliated and nonpiliated strains with time, with maximum adherence occurring between 24-36 h. Immunofluorescence and electron microscopic assays visually confirmed the radioactive adherence findings. These results characterize the kinetics of Hib adherence to BEC. BEC incubated with nonpiliated $\mathrm{Hib}$ for $30 \mathrm{~h}$ were found to be coated with piliated strains, suggesting the induction of pilus production or the selection of a piliated subpopulation. (Pediatr Res 26:500-503, 1989)
\end{abstract}

\section{Abbreviations}

BEC, buccal epithelial cells

BHI, brain heart infusion

CFU, colony-forming unit

CSF, cerebrospinal fluid

EM, electron microscopy

Hib, Haemophilus influenzae type b

${ }^{3} \mathrm{H}$-Hib, tritium-labeled Hib

IF, immunofluorescent

LOS, lipooligosaccharide

NP, nasopharyngeal

$\mathrm{P}+$, piliated

$P-$, nonpiliated

PRP, polyribosylribitol phosphate

Hib is the leading cause of bacterial neningitis in the United States (1). An infant rat model for Hib meningitis has delineated four separate, sequential steps in the pathogenesis consisting of

Received March 7, 1989; accepted June 27, 1989.

Correspondence Christian C. Patrick, M.D., Ph.D, St. Jude Children's Research Hospital, Department of Infectious Diseases, 332 N. Lauderdale, P. O. Box 318 , Memphis, TN 38101

${ }^{1}$ Presented in part at the ASM Annual Meeting, Miami Beach, May 1988.

${ }^{2}$ Recipient of the Speros P. Martel Fellowship Award at Texas Children's Hospital.
1) naso/oropharyngeal colonization, 2) penetration of the mucosa with entry into the bloodstream, 3) development of a bacteremia, and 4) development of meningitis $(2,3)$.

The colonization of the naso/oropharyngeal area is considered to be the initial step in the pathogenesis of Hib infections (4). The adherence of Hib to naso/oropharyngeal cells for colonization has not been clearly defined. There are conflicting lines of evidence as to the contribution pili or fimbriae have in this process. In vitro, $\mathrm{P}+\mathrm{Hib}$ have an increased adherence to erythrocytes (5), BEC (6), and pharyngeal cells (7), but the majority of NP isolates of $\mathrm{Hib}$ from patients with systemic diseases at the time of hospital admission are $\mathrm{P}-$, and all blood and CSF isolates are $\mathrm{P}-(8)$. Additionally, $\mathrm{P}+$ and $\mathrm{P}-\mathrm{Hib}$ are equally virulent in the infant rat model (9). This study further characterizes the kinetics of $\mathrm{Hib}$ adherence for $\mathrm{P}+$ and $\mathrm{P}-$ isolates to $\mathrm{BEC}$.

\section{MATERIALS AND METHODS}

Bacterial Strains and Media. Hib isolates used in this study have been previously described (8). Three paired isolates were studied, 1007,$1009 ; 880,884 ; 1228,1264$ consisting of a CSF $\mathrm{P}-$ isolate and a NP P+ isolate, respectively. EM was used to confirm the presence or absence of pili (8).

Hib were grown in BHI broth supplemented with $10 \mu \mathrm{g} / \mathrm{mL}$ of NAD and Hemin (Sigma Chemical Company, St. Louis, MO) in $5 \% \mathrm{CO}_{2}$ at $37^{\circ} \mathrm{C}$. Isolates were not serially passaged but were frozen at $-70^{\circ} \mathrm{C}$ in $\mathrm{BHI}$ with $10 \%$ glycerol, $\mathrm{pH} 7.3$.

$\mathrm{Hib}$ were labeled for radioactive adherence assay by inoculating five colonies of $\mathrm{Hib}$ into $10 \mathrm{~mL}$ of BHI supplemented with NAD and Hemin with $25 \mu \mathrm{Ci}$ tritium-labeled amino acids (1 $\mu \mathrm{Ci} / \mu \mathrm{L}$; TRK 440 Amersham Corp., Arlington Heights, IL) overnight. The ${ }^{3} \mathrm{H}-\mathrm{Hib}$ were harvested by centrifugation at 7000 $\times g$ for 10 min at $4^{\circ} \mathrm{C}$ and washed three times in Dulbecco's PBS, pH 7.3. The specific activity was $1.5 \times 10^{-4}$ to $5.5 \times 10^{-5}$ $\mathrm{cpm} / \mathrm{CFU}$. The organisms were resuspended in Dulbecco's PBS, $\mathrm{pH} 7.3$ with $1 \%$ gelatin (Sigma).

Eucaryotic Cells. BEC were obtained from healthy male volunteers and handled in a manner previously described (10). A final suspension of $10^{4} \mathrm{BEC} / \mathrm{mL}$ was obtained by counting using a hemocytometer. Cell viability was assessed by adding $0.9 \mathrm{~mL}$ trypan blue (GIBCO Laboratories, Grand Island, NY) with 0.1 $\mathrm{mL}$ of eucaryotic cells and counting 100 cells with a hemocytometer.

Adherence Assays. Radioactive assay. A total of $0.5 \mathrm{~mL}$ of ${ }^{3} \mathrm{H}$ $\mathrm{Hib}$ at $10^{8} \mathrm{CFU} / \mathrm{mL}$ was incubated with $0.5 \mathrm{~mL}$ of BEC $\left(10^{4}\right.$ cells $/ \mathrm{mL}$ ) for varying time periods at $37^{\circ} \mathrm{C}$ in a reciprocal water bath. Colony counts were determined for each experiment. Nonadherent ${ }^{3} \mathrm{H}-\mathrm{Hib}$ were removed by washing cells three times with Dulbecco's PBS, pH 7.3 using a Millipore manifold system (Millipore Corporation, Bedford, MA) with 12- $\mu \mathrm{m}$ Nucleopore polycarbonate filters (Nucleopore, Pleasanton, CA). The filters 
were removed from the manifold and placed into scintillation vials with $10 \mathrm{~mL}$ of scintillation fluid (Scintiverse II, Fisher Scientific, Orangeburg, NY). Duplicate vials were counted in a scintillation counter for 2 min.

Controls consisted of 1$)^{3} \mathrm{H}$-Hib alone without BEC and 2) a control for the spontaneous release of ${ }^{3} \mathrm{H}$ by ${ }^{3} \mathrm{H}$-Hib with subsequent uptake by BEC (radio-release control). Total $\mathrm{cpm}$ for ${ }^{3} \mathrm{H}-$ Hib was obtained by placing $100 \mu \mathrm{L}$ of washed ${ }^{3} \mathrm{H}-\mathrm{Hib}$ into a scintillation vial, counting as described above, and multiplying by 5 to give total cpm per $0.5 \mathrm{~mL}$.

Briefly, the radio-release control involved incubating the ${ }^{3} \mathrm{H}$ Hib $\left(10^{8} \mathrm{cfu} / \mathrm{mL}\right)$ without BEC for identical time periods as the adherence assay. The ${ }^{3} \mathrm{H}$-Hib were then removed by a filtration using a $0.22-\mu \mathrm{m}$ syringe filter (Millipore Corporation). The filtrate was then incubated with fresh BEC $\left(10^{4}\right.$ cells $\left./ \mathrm{mL}\right)$ for identical incubation periods as the adherence assay and handled as described above for radioactive counting.

An adherence percentage was calculated using the following formula:

$$
\frac{\begin{array}{r}
\left(\mathrm{cpm} \text { of } \mathrm{BEC}+{ }^{3} \mathrm{H}-\mathrm{Hib}\right)-\left(\mathrm{cpm}{ }^{3} \mathrm{H}-\mathrm{Hib} \text { alone }\right) \\
-(\mathrm{cpm} \text { of radio-release control })
\end{array}}{\text { total } \mathrm{cpm} \text { of }{ }^{3} \mathrm{H}-\mathrm{Hib}}
$$

IF adherence assay. The indirect IF adherence assay was identical to the radioactive adherence assay except the Hib were not radiolabeled. After incubation for varying times, the bound Hib to BEC were removed from the unbound Hib using the Millipore manifold system with Nucleopore filters as described. The cells were displaced from the filters with gentle agitation into Dulbecco's PBS, pH 7.3, and subsequently spun onto glass slides using a cytospin centrifuge. Controls consisted of Hib and BEC alone, or BEC plus an Hib strain known to adhere by EM studies. The slides were air-dried and fixed in ethanol. Indirect IF was achieved by incubating the slide for $60 \mathrm{~min}$ in a moist chamber at room temperature with an anti-Hib LOS murine MAb kindly provided by Dr. Eric J. Hansen, University of Texas Health Science Center at Dallas as the first step. Anti-Hib LOS was used instead of anti-whole Hib antisera containing predominately anticapsular PRP antibody because of the difficulty of released capsular PRP during incubation periods as previously described by Anderson et al. (11). The released PRP coated the $\mathrm{BEC}$, increasing the background intensity, rendering fluorescent visualization very difficult. This problem was overcome by using an anti-LOS MAb which was known to be specific for the LOSserotype (12) of the isolates studied. This MAb has been described elsewhere (13). After incubation with anti-LOS MAb the slides were washed and incubated with fluorescein-conjugated goat antimouse $\mathrm{IgG}$ (Sigma) for $30 \mathrm{~min}$ in a moist chamber. The slides were again washed and counterstained with $0.2 \%$ Evans blue dye for $2 \mathrm{~min}$, allowed to air dry in the dark and examined by oil immersion with a fluorescence microscope. The number of adherent bacteria per BEC were quantitated for 25-50 eucaryotic cells.

$E M$ adherence assay. The assay system was identical to the indirect IF adherence assay. The nonadherent Hib were again removed using Nucleopore filters. Adherent Hib to BEC were fixed in 3\% phosphate-buffered glutaraldehyde and subjected to critical-point drying with carbon dioxide. The samples were then coated with 150 angstroms $(15 \mathrm{~nm}$ ) of gold pallidium in a Denton vacuum evaporator. Specimens were viewed with a Joel 100/cx electron microscope. The number of adherent bacteria per BEC was assessed counting 50 eucaryotic cells.

Statistical Analysis. Student's $t$ test analysis was used for statistical analysis of continuous variables. Proportions were analyzed by $\chi^{2}$.

\section{RESULTS}

Three paired isolates from children with $\mathrm{Hib}$ meningitis were used for adherence kinetics using BEC. One set of isolates (1007 and 1009) was studied in depth. All paired isolates consisted of a NP isolate which was predominately $(>98 \%) \mathrm{P}+$ and a CSF isolate which was predominately $(>99 \%) \mathrm{P}-$. The presence or absence of pili was assessed using EM (data not shown).

Figure 1 shows a kinetic analysis of the two prototypic isolates, $1007(\mathrm{P}-)$ and $1009(\mathrm{P}+)$ adhering to $\mathrm{BEC}$ as assessed by percent radioactive binding. The piliated isolate, 1009 , had significantly higher percent radioactive binding to $\mathrm{BEC}$ than the $\mathrm{P}-$ isolate $(p=0.002)$. Minimal binding was noted at $1 \mathrm{~h}$ for $1007(\mathrm{P}-)$ adhering to BEC. Both Hib isolates showed increasing adherence to BEC with time with maximum binding occurring at $36 \mathrm{~h}$. Quantitative studies measuring bacterial CFU obtained during this time study showed a maximum 2-log death rate over $48 \mathrm{~h}$. Bacterial contamination from normal oral flora became apparent at 36-48 $\mathrm{h}$ with $\mathrm{BEC}$ adherence. No appreciable change in viability of $\mathrm{BEC}$ with time was evident using trypan blue studies. Thus, a 30-h incubation was chosen as a prolonged time point to compare to the standard $1-\mathrm{h}$ incubation for time kinetic studies.

Tablel shows percent radioactive binding of the two prototypic Hib isolates with BEC at 1- and 30-h incubation, 1007 (P-) and $1009(\mathrm{P}+)$ bound to $\mathrm{BEC}$ at $30 \mathrm{~h}$ significantly more when compared to $1 \mathrm{~h}$ binding values $(p \leq 0.002)$. Of note is the moderate degree of adherence by the non-piliated isolate, 1007 to BEC cells at $30 \mathrm{~h}$.

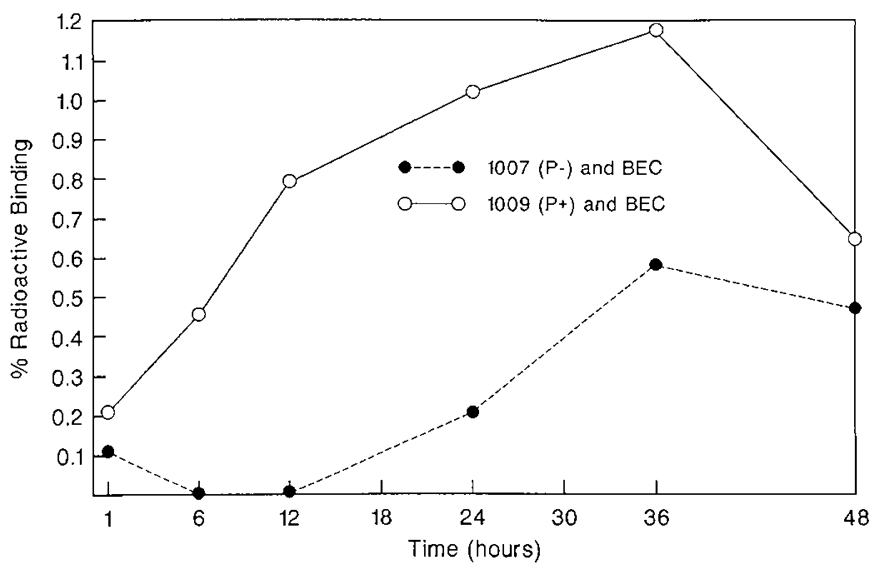

Fig. 1. Kinetic analysis of percent radioactive binding of prototypic $\mathrm{P}+(1009)$ and $\mathrm{P}-(1007)$ Hib to BEC.

Table 1. Time kinetic adherence as assessed by radioactive binding assay for prototypic piliated (1009) and nonpiliated (1007) Hib strains to BEC

\begin{tabular}{cccr}
\hline & \multicolumn{2}{c}{$\%$ Radioactive Binding } & \\
\cline { 2 - 3 } Strain & $1 \mathrm{H}$ & \multicolumn{1}{c}{$30 \mathrm{H}$} & \multicolumn{1}{c}{$p$} \\
\hline $1007(\mathrm{P}-)$ & $0.003 \pm 0.008^{*}(6) \dagger$ & $0.42 \pm 0.1(9)$ & $<0.001$ \\
$1009(\mathrm{P}+)$ & $0.16 \pm 0.06(4)$ & $1.83 \pm 0.78(6)$ & 0.002 \\
\hline
\end{tabular}

$*$ Mean \pm SD.

$\dagger$ Number of experiments, each in duplicate in parentheses.

\begin{tabular}{|c|c|c|c|}
\hline \multirow[b]{2}{*}{ Strain } & \multicolumn{2}{|c|}{$\%$ Radioactive binding } & \multirow[b]{2}{*}{$p$} \\
\hline & $1 \mathrm{H}$ & $30 \mathrm{H}$ & \\
\hline $880(\mathrm{P}-)$ & 0 & $2.03 \pm 0.80 \dagger$ & $<0.001$ \\
\hline $884(\mathrm{P}+)$ & $0.94 \pm 0.13$ & $2.57 \pm 0.70$ & 0.0004 \\
\hline $1228(\mathrm{P}-)$ & 0 & $0.70 \pm 0.16$ & $<0.001$ \\
\hline $1264(\mathrm{P}+)$ & $0.22 \pm 0.08$ & $1.13 \pm 0.89$ & 0.06 \\
\hline
\end{tabular}

Table 2. Time kinetic adherence as assessed by radioactive binding for paired Hib strains to $B E C^{*}$

* Results of two experiments, each in duplicate.

$\dagger$ Mean \pm SD. 
Complementary data using two additional paired isolates are shown in Table 2 . No adherence was detected at $1 \mathrm{~h}$ except for piliated isolates $(884,1264)$ binding to $\mathrm{BEC}$ cells. All isolates adhered to BEC at $30 \mathrm{~h}$; adherence in all cases but one (1264 to $\mathrm{BEC}$ ) was significantly increased at $30 \mathrm{~h}$ compared to binding at $1 \mathrm{~h}$, regardless of the initial state of piliation.

Indirect IF and EM adherence assays were used to visualize adherence independent of the radioactive adherence data. Table 3 shows the percentage of BEC with different numbers of adherent Hib per eucaryotic cell at 1 and $30 \mathrm{~h}$ for the two prototypic

Table 3. Adherence of Hib to BEC: representative indirect immunofluorescent and electron microscopic studies

\begin{tabular}{lrrrrrr}
\hline & \multicolumn{6}{c}{$\begin{array}{c}\text { No. of adherent bacteria/cell } \\
\text { Incubation time }\end{array}$} \\
\cline { 2 - 7 } & \multicolumn{3}{c}{$1 \mathrm{H}$} & \multicolumn{5}{c}{$30 \mathrm{H}$} \\
\hline $\begin{array}{l}\text { Immunofluores- } \\
\text { cence }\end{array}$ & 0 & $1-5$ & $>5$ & 0 & $1-5$ & $>5$ \\
$\quad 1007(\mathrm{P}-)$ & $92^{*}$ & 8 & 0 & 52 & 48 & 0 \\
$1009(\mathrm{P}+)$ & 20 & 32 & 48 & 0 & 28 & 72 \\
& & & & & & \\
Electron microscopy & & & & & & \\
$1007(\mathrm{P}-)$ & 84 & 12 & 4 & 0 & 16 & 84 \\
$1009(\mathrm{P}+)$ & 8 & 30 & 62 & 2 & 2 & 96 \\
\hline
\end{tabular}

* Percentage of buccal epithelial cells with the indicated number of adherent Hib. $p$ values for all comparisons ( 1 versus $30 \mathrm{~h}$ ) $p<0.001$.
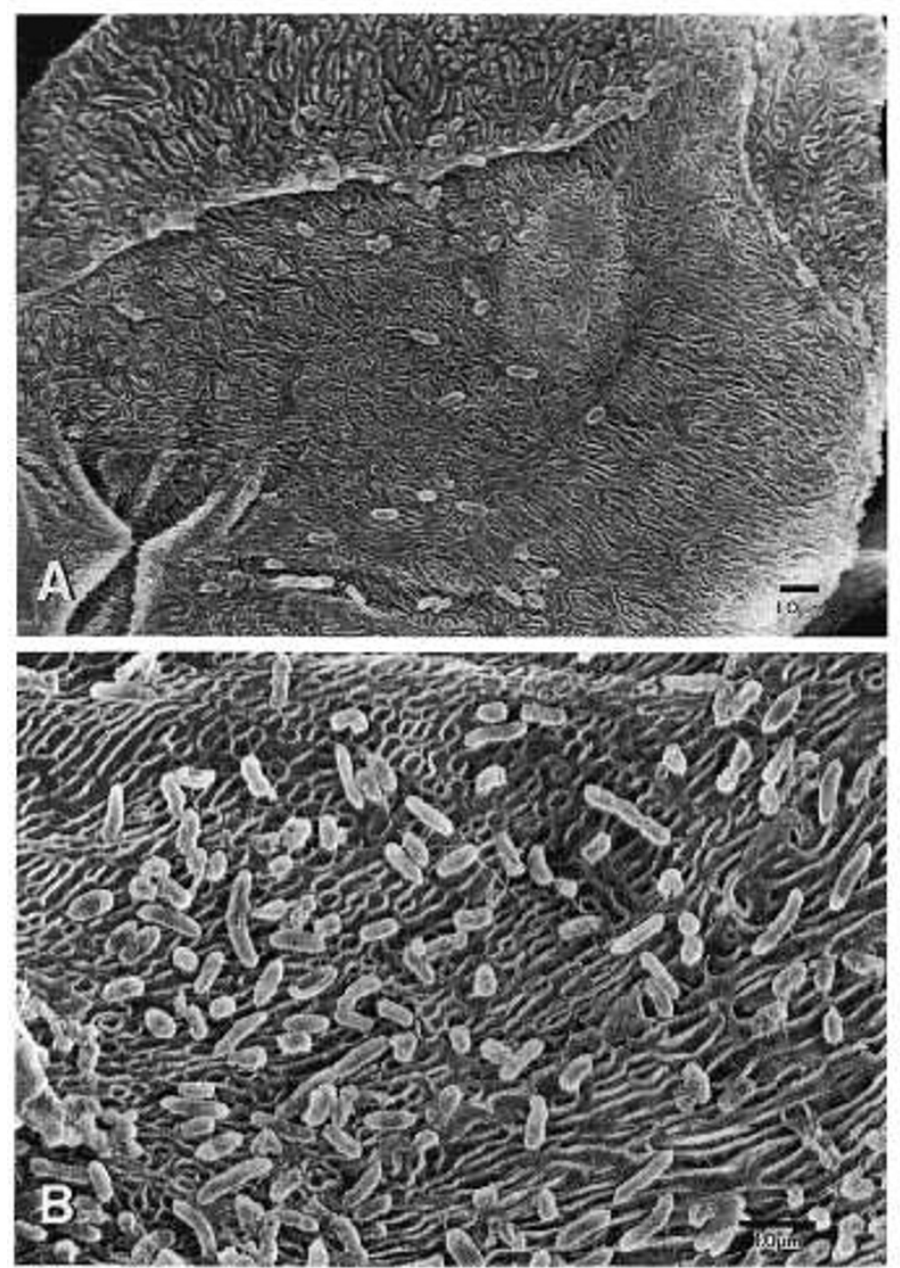

Fig. 2. EM photomicrographs of prototypic Hib isolate 1009 adhering to buccal epithelial cells at $1 \mathrm{~h}(A, 3000 \times)$ and at $30 \mathrm{~h}(B, 6000 \times)$ incubations. isolates. Small numbers of $1007(\mathrm{P}-)$ could be seen adhering to $\mathrm{BEC}$ at $1 \mathrm{~h}$. The piliated Hib isolate (1009) adhered well at $1 \mathrm{~h}$ both with the indirect IF and EM studies. At $30 \mathrm{~h}$ all isolates adhered in significantly greater numbers to BEC compared to 1 $h$ values confirming the radioactive assay.

Figure $2 A$ shows EM photomicrographs of the piliated prototypic Hib isolate (1009) with more than 5 bacteria bound to this $\mathrm{BEC}$ at $1 \mathrm{~h}$. Figure $2 B$ shows this same strain, 1009 binding to a BEC cell at $30-\mathrm{h}$ incubation with an appreciable increase in bound bacteria. The $\mathrm{P}$ isolate, 1007 , had minimal binding to $\mathrm{BEC}$ at $1 \mathrm{~h}$ (figure not shown), but at $30 \mathrm{~h}$ the majority $(84 \%)$ of $\mathrm{BEC}$ had $>5$ bacteria adhering to them (Fig. $3 A$ ). The presence of pili on the adherent bacteria became apparent upon increasing the magnification to $30000 \times$ (Fig. $3 B$ ).

\section{DISCUSSION}

The ability of Hib to adhere to the naso/oropharyngeal epithelia is the presumed initial step in pathogenesis of Hib infections. Pili or fimbriae discovered on Hib in $1982(6,7)$ were originally thought to be the sole mediators of Hib adherence in a manner similar to Enterobacteriaceae adherence (14). Recent evidence has suggested the presence of a second $\mathrm{Hib}$ adhesin in addition to pili $(15,16)$. The significance of these two adhesins is not known. This study was designed to evaluate the role of pili in adherence with time because 1 ) $\mathrm{P}-\mathrm{Hib}$ that do not adhere to $\mathrm{BEC}$ in vitro are the predominant organisms found in the nasopharynx in patients with systemic disease (8), and 2) the mucous
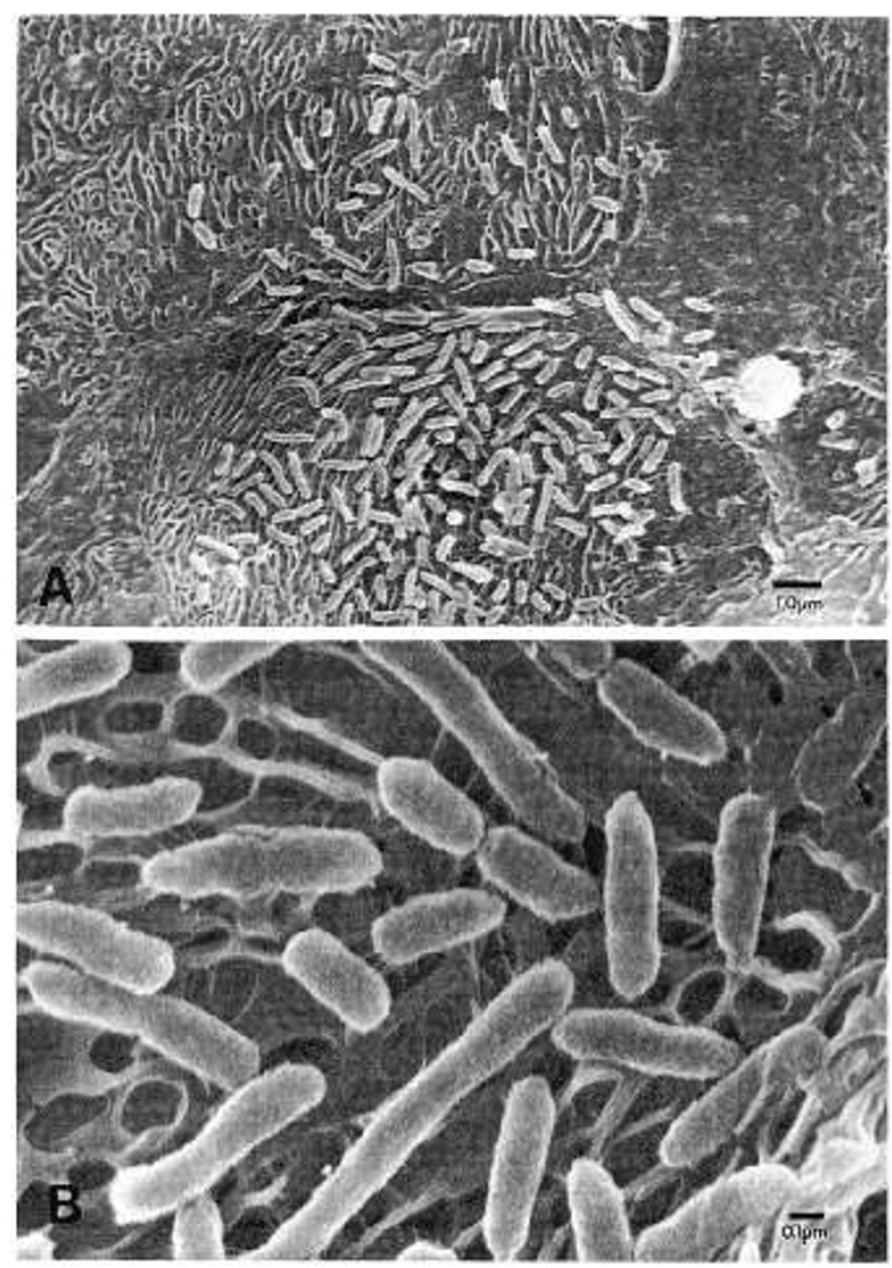

Fig. 3. EM photomicrographs of prototypic Hib isolate 1007 adhering to $\mathrm{BEC}$ at 30 -h incubation $(A, 4000 \times) . B$ shows higher magnification of Hib isolate 1007 adhering to $\mathrm{BEC}$ with apparent pili $(30000 \times)$. 
blanket which coats the NP region (17) could keep the Hib in close proximity to eucaryotic cells for prolonged intervals.

We studied $\mathrm{P}+$ and $\mathrm{P}-\mathrm{Hib}$ adherence to $\mathrm{BEC}$ with three paired Hib clinical isolates over a 30 -h time period. The conditions of our assay were kept similar to previous studies to compare time-course analysis. A ratio of bacteria to eucaryotic cells of $10^{4}: 1$ was chosen because other adherence model systems $(18,19)$ and the Hib system $(10,20)$ used this ratio. The temperature of $37^{\circ} \mathrm{C}$ and $\mathrm{pH}$ of 7.3 were kept constant as previously described (10). Additionally, one percent gelatin was included in our assay system to minimize non-specific binding to Nucleopore filters (9).

Kinetic analyses were evaluated for $\mathrm{P}+$ and $\mathrm{P}-$ mediated adherence. Previous work by Lampe et al. (10) and Pichichero (20) had shown little change in adherence with epithelial cells after $30 \mathrm{~min}$ of incubation, although the longest incubation time was $2 \mathrm{~h}$ in the Lampe et al. (10) study.

In this study, all three modalities used to assess adherence reconfirmed binding of piliated $\mathrm{Hib}$ to $\mathrm{BEC}$ at $1 \mathrm{~h}$. Timed adherence experiments showed a significant increase in adherence with time which has not been previously reported. The reason for this increased adherence is not known but could be due to an increase in exposed pili receptors on BEC, an increase in the number of pili on $\mathrm{Hib}$, or an increase in exposure time allowing more $\mathrm{Hib}$ to adhere to $\mathrm{BEC}$. The $\mathrm{P}-$ strains bound minimally or not at all to BEC at $1 \mathrm{~h}$ which is consistent with their being $>99 \% \mathrm{P}-$; incubation to $30 \mathrm{~h}$ produced pronounced adherence of the $\mathrm{P}-$ strain to BEC in all three assay systems. EM studies showed pili on the attached $\mathrm{Hib}$ which were more than $99 \% \mathrm{P}-$ when first incubated with the BEC. This increased binding with time by the $\mathrm{P}-\mathrm{Hib}$ appears to be occurring by the proposed high frequency switching of the $\mathrm{P}-\mathrm{Hib}$ to a $\mathrm{P}+$ form (5).

We have shown that the adherence of both $\mathrm{P}+$ and $\mathrm{P}-\mathrm{Hib}$ to $\mathrm{BEC}$ increases as the incubation period increases. EM data have shown that the P- Hib have demonstrable pili on the surface when bound to $\mathrm{BEC}$ at $30 \mathrm{~h}$ indicating that Hib could modify their state of piliation to achieve adherence.

\section{REFERENCES}

1. Geiseler PJ, Nelson KE, Levin S, Reddi KT, Moses VK 1980 Community acquired purulent meningitis: a review of 1316 cases during the antibiotic era 1954-1976. Rev Infect Dis 2:725-745
2. Moxon ER, Smith AL, Averill DR, Smith DH 1974 Haemophilus influenzae meningitis in infant rats after intranasal inoculation. J Infect Dis 129:154162

3. Turk DC 1984 The pathogenicity of Haemophilus influenzae. J Med Micro 18:1-16

4. Ostrow PT, Moxon ER, Vernon N, Kapko R 1979 Pathogenesis of bacterial meningitis. Studies on the route of meningeal invasion following Haemophilus influenzae inoculation of infant rats. Lab Invest 40:678-685

5. Connor EM $\mathrm{J}_{\mathrm{r}}$, Loeb MR 1983 A hemadsorption method for detection of colonies of Haemophilus influenzae type b expressing fimbriae. J Infect Dis 148:855-860

6. Pichichero ME, Loeb M, Anderson P, Smith DH 1982 Do pili play a role in pathogenicity of Haemophilus influenzae type b? Lancet 2:960-962

7. Guerina NG, Langermann S, Clegg HW, Kessler TW, Goldmann DA, Gilsdor JR 1982 Adherence of piliated Haemophilus influenzae type b to oropharyngeal cells. J Infect Dis 146:564

8. Mason EO Jr, Kaplan SL, Wiedermann BL, Norrod EP, Stenback WA 1985 Frequency and properties of naturally occurring adherent piliated strains of Haemophilus influenzae type b. Infect Immun 49:98-103

9. Kaplan SL, Mason EO Jr, Wiedermann BL 1983 Role of adherence in pathogenesis of Haemophilus influenzae type $\mathrm{b}$ infection in infant rat. Infect Immun 42:612-617

10. Lampe RM, Mason Jr EO, Kaplan SL, Umstead CL, Yow MD, Feigin RD 1982 Adherence of Haemophilus influenzae to buccal epithelial cells. Infect Immun 35:166-172

11. Anderson P, Pitt J, Smith DH 1976 Synthesis and release of polyribophosphate by Haemophilus influenzae type b in vitro. Infect Immun 13:581-589

12. Gulig PA, Hansen EJ 1985 Coprecipitation of lipopolysaccharide and the 39,000-molecular-weight major outer membrane protein of Haemophilus influenzae type b by lipopolysaccharide-directed monoclonal antibody. Infect Immun 49:819-827

13. Gulig PA, Patrick CC, Hermanstorfer L, McCracken GH Jr, Hansen EJ 1987 Conservation of epitopes in the oligosaccharide portion of the lipooligosaccharide of Haemophilus influenzae type b. Infect Immun 55:513-520

14. Beachey EH 1981 Bacterial adherence: adhesin-receptor interactions mediating the attachment of bacteria to mucosa surfaces. J Infect Dis 143:325-345

15. Sable NS, Connor EM, Hall CB, Loeb MR 1985 Variable adherence of fimbriated Haemophilus influenzae type b to human cells. Infect Immun 48:119-123

16. Loeb MR, Connor E, Penney D 1988 A comparison of the adherence of fimbriated and nonfimbriated Haemophilus influenzae type $\mathrm{b}$ to human adenoids in organ culture. Infect Immun 56:484-489

17. Jones GW, Isaacson RE 1983 Proteinaceous bacterial adhesins and their receptors. Crit Rev Microbiol 10:229-260

18. Anderson B, Eriksson B, Falsen E, Fogh A, Hanson LA, Nylen O, Peterson $\mathrm{H}$ Svanborg-Eden C 1981 Adhesion of Streptococcus pneumoniae to human pharyngeal epithelial cells in vitro: differences in adhesive capacity among strains isolated from subjects with otitis media, septicemia or meningitis or from healthy carriers. Infect Immun 32:311-317

19. Svanborn-Eden C, Eriksson B, Hanson LA 1977 Adhesion of Escherichia coli to human uroepithelial cells in vitro. Infect Immun 18:767-774

20. Pichichero ME 1984 Adherence of Haemophilus influenzae to human buccal and pharyngeal epithelial cells: relationship to pilation. J Med Microbiol 18:107-116 